\title{
Improvement of attention span and reaction time with hyperbaric oxygen treatment in patients with toxic injury due to mold exposure
}

\author{
N. Ezra $\cdot$ K. Dang $\cdot$ G. Heuser
}

Received: 29 November 2009 / Accepted: 3 April 2010 /Published online: 27 October 2010

(C) The Author(s) 2010. This article is published with open access at Springerlink.com

\begin{abstract}
It is, by now, well established that mold toxins (mycotoxins) can cause significant adverse health effects. In this study, 15 subjects who developed an attention deficit disorder (ADD) and slowing of reaction time at the time of exposure to mold toxins were identified. Deficits in attention span and reaction time were documented not only by taking a careful history, but also by performing a Test of Variables of Attention (TOVA). The TOVA test provides an objective measure of these two variables. It was found that moldexposed subjects show statistically significant decreases in attention span and significant increases in reaction time to stimuli compared to controls. After ten sessions of hyperbaric oxygen treatment (HBOT), a statistically significant improvement was seen in both measures. This preliminary study suggests promising outcomes in treating mold-exposed patients with hyperbaric oxygen.
\end{abstract}

\section{Introduction}

Toxic chemical exposure such as mold and mycotoxins can significantly affect brain function in children and adults. Mycotoxins are secondary metabolites of fungi, usually molds, which can cause injury to animals or humans [1]. It

This paper was presented as a poster presentation at the UCLA Science Poster Day in Los Angeles, CA, in May 2005 and 2006.

\footnotetext{
N. Ezra $\cdot$ K. Dang $\cdot$ G. Heuser

Department of Medicine,

David Geffen School of Medicine at UCLA,

Los Angeles, CA, USA

G. Heuser $(\bowtie)$

923 Laguna Street, Suite B,

Santa Barbara, CA 93101, USA

e-mail: gheuser@ucla.edu
}

has been shown that these effects may last for years after toxic exposure has ceased [2].

Exposure to toxins, including mold, can lead to significant long-lasting impairment of brain function, often inducing temporary to permanent disability. Typical complaints include impairment of short-term memory, intermittent disorientation, disturbed balance and coordination, inability to multi-task, and impairment of attention span and reaction time. Sick building syndrome (SBS) may be caused by exposure to mold and mold toxins. Symptoms of SBS, a widely described but poorly understood constellation of symptoms that has been observed in certain persons in "sick" buildings, include headaches, poor concentration, fatigue, memory loss, dry skin, and itchy eyes $[3,4]$. This is seen in adults as well as young people.

Toxic opportunistic molds include: Stachybotrys chartarum, Aspergillus species (A. fumigatus, A. flavus, A. niger, A. versicolor, etc.), Cladosporium, Alternaria, Penicillium, Trichoderma, Fusarium graminearum, etc. [5]. These molds flourish in moist and damp environments and may lead to a number of mycotoxicoses, a collective name for all of the diseases caused by toxic molds [5].

After investigating the health complaints of 209 patients with confirmed exposure to mixed mold infestation, a recent study concluded that exposure to mixed molds and their associated mycotoxins in water-damaged buildings leads to multiple health problems involving the central nervous system and the pulmonary system [6]. Patients typically display signs of pathology, including headaches, gastrointestinal and neuromuscular abnormalities, and various other complaints [7].

Objective techniques such as brainstem auditory evoked response (BAER) tests have indicated abnormalities in patients exposed to toxic opportunistic molds through indoor air contamination [5]. Clinical studies carried out 
on children with a history of mold exposure, involving a series of neurophysiologic tests such as electroencephalogram (EEG), brainstem evoked potential (BAEP), visual evoked potential (VEP), and somatosensory evoked potential (SSEP), rendered abnormal results, indicating significant neurological defects and revealing the extent to which toxic molds can neurologically and behaviorally affect children [8]. There is evidence of diffuse polyneuropathy and slow motor conduction, indicating significant neurological deficits in all patients with exposure to toxic molds [8]. Myconeurotoxicity may result from effects of the mycotoxins acting directly on the elements of the nervous system or acting on other biological systems, which then adversely affect the nervous system [9].

In many of the mold-exposed patients in question, attention span and reaction time have become impaired. Attention deficit disorder (ADD) is typically diagnosed by taking a full history complemented with psychometric testing. Although most diagnoses occur during adolescence, ADD can occur in adults [10].

Having previously found that functional brain scans (SPECT) are abnormal in mold-exposed patients and improve after hyperbaric oxygen treatment (HBOT) [11], it was unknown whether the Test of Variables of Attention (TOVA) could also improve after HBOT. A recent pilot study showed improvement of TOVA after HBOT. However, this study was too small to allow for statistical analysis, having been implemented in only several subjects, as opposed to the current study, which includes 15 adults.

\section{Materials and methods}

\section{Subjects}

Fifteen adults (ages 18 to 58 years) were entered into the study after it was established that they had been exposed to mold, mycotoxins, and other related byproducts and had become ill as a result. The subjects were recruited by direct referrals to the corresponding author from practicing physicians, and out of those, only subjects confirmed to have been exposed to mold were submitted to the study. Participants consented to participate in the research study. The UCLA IRB approved the consent forms and this study. Although subjects were not specifically evaluated for depression or other cognitive deficiencies, symptoms of such disorders were not apparent during the clinical evaluation. All potential candidates were entered into the experimental group with the ages of those chosen for the sample size following a fairly normal distribution. The testing of the patients' residence, work site, and school (in the case of exposed students) confirmed the presence of mold and mycotoxins upon evaluation.
Exposure and immune reaction to mold and mold toxins was established by performing specific antibody studies (to individual molds and mold toxins). Establishing that subjects possessed adverse health effects due to mold exposure involved using antibodies against molds and mycotoxins. Subjects were referred for immunological evaluation and were confirmed to have mold antibodies in greater concentration than in healthy blood donors [12]. Since the antibodies studied were concluded to be explicit to mold antigens and mycotoxins, they were understandably used as indicators for toxin-exposed patients eligible to be entered into the study.

Ill effects from exposure were measured with objective tests, including functional brain scans (SPECT) [13], pulmonary function tests, etc. One of the tests commonly used is the TOVA [14], a computerized simulation which can be done in the office. With the research advisor having seen more than 500 patients with a history of mold exposure in recent years, we noticed that many had developed a clinical ADD which was confirmed by an abnormal TOVA. TOVA tests were administered in a quiet setting with the examiner observing the patient during the session. The tests were repeated after HBOT had been given for ten consecutive (except for weekends) days. All TOVA tests were done during the morning hours.

TOVA measures include the variability of response time (consistency), response time, commission (impulsivity), errors of omission (inattention), post-commission response times, multiple and anticipatory responses, and an attention deficit hyperactivity disorder (ADHD) score, which is a comparison to an age/gender-specific ADHD group. A compilation of the above results is calculated and called the $\mathrm{D}$ prime score that provides an overall assessment of the subject.

The TOVA is composed of a 21.6-min-long, very simple "computer game" that measures the subject's responses to visual stimuli. These measurements are then compared to the measurements of a group of people without attention disorders who took the TOVA and a printout of the results is generated by the program. The TOVA is extensively normed for both children and adults (ages 4 to $80+$ years). It precisely measures reaction times $( \pm 1 \mathrm{~ms})$ and it is language- and culture-free.

The TOVA uses geometric stimuli to minimize the effects of cultural differences and learning problems, and contains two test conditions: target infrequent and target frequent. When a subject responds to the nontarget, it is called an error of commission and is a measure of impulsivity. Thus, the ability to pay attention to a boring, repetitive task is best measured in the first half of the TOVA, while the ability to inhibit oneself is best measured in the second half.

The TOVA uses a fixed, mid-range interstimulus interval (2 s) and visual stimuli. The stimuli are nonsequential, simple geometric configurations and monochromatic. Since these features, along with the use of a 2.5 -min practice 
round, minimize practice effects, the TOVA can be used for serial measurements.

All subjects agreed to have their results published and provided consent to be treated by hyperbaric oxygen, having been informed of any potential side effects. Statistical analysis of TOVA results was performed by using a paired samples $t$-test via the Statistical Package for the Social Sciences (SPSS) software program.

\section{Treatment protocol}

HBOT was administered in a portable chamber into which air was delivered to a pressure reading of 1.3 ATM. The pressure was gently increased to the above value over about $15 \mathrm{~min}$ after the patient had entered the chamber. One hundred percent oxygen was delivered into the chamber and was measured to have a concentration of $24-34 \%$ after it had been diluted in the chamber air. Oxygen was not delivered by mask or nasal canals, allowing the patient to move freely about the chamber. The patient remained in the chamber for $1 \mathrm{~h}$, after which the pressure was gently decreased before the patient exited the chamber.

The treatment protocol shown in Table 1 illustrates the differences from traditional HBOT. Because both pressure and oxygen are lower than in traditional chambers, the term "mild HBOT" (mHBOT) has been used. Treatments were rendered once or twice a day with approximately $4 \mathrm{~h}$ separating the treatments on a given day. All patients in this study were treated with ten consecutive daily treatments )except on weekends_. Patients continued on whatever medical treatment they had been on and, therefore, no new treatment modality was introduced during mHBOT.

\section{Results}

After ten treatment sessions of mHBOT, the preliminary results showed that the 15 individuals in the study

Table 1 Portable hyperbaric oxygen chambers were used as a form of mild hyperbaric oxygen treatment (mHBOT) with the given conditions compared to traditional HBOT. Atmosphere absolute (ATA) of 1 ATA is the atmospheric pressure at sea level measured with a barometer. mHBOT uses 1.3 ATA compared to 2-3 ATA in traditional chambers. Pound per square inch gauge (PSIG) is the pressure referenced to ambient air pressure indicated by a pressure gauge using atmospheric pressure as a base. This value in mHBOT, 4.7, is much significantly improved in attention span, reaction time, consistency, and an overall D prime score assessed via the TOVA.

The effect of ten treatments of mHBOT on patient inattention, reaction time, variability, and $\mathrm{D}$ prime score as tested by the TOVA in subjects with mold exposure is illustrated in Fig. 1.

To test whether the average attention span was higher in the after mHBOT condition $(\mathrm{M}=96.47, \mathrm{SD}=21.11)$ than in the before mHBOT condition $(\mathrm{M}=72.67, \mathrm{SD}=32.35)$, the means of these two conditions were compared using a paired samples $t$-test. This analysis revealed that, on average, participants significantly improved their attention span after ten mHBOT sessions, $t(14)=3.55, p<0.05$. The same test was used to analyze the change in scores of reaction time $[t(14)=4.41]$, consistency $[t(14)=4.82]$, and overall D prime score $[t(14)=3.58]$, and showed a $p<0.05$ value for all categories.

\section{Discussion}

While ADD is usually thought of as a disorder in the young, it can certainly occur in adults. In this case, it should be considered as acquired, i.e., secondary to toxic exposure. ADHD in adults is reasoned to be similar in nature to the commonly known childhood disorder with regard to cognitive and psychiatric findings [15].

In recent years, there have been an increasing number of patients who were exposed to mold and mold toxins. Many of these patients complain of impaired brain function and turn out to have abnormal neuropsychological tests, abnormal SPECT brain scans, and abnormal TOVA tests. The TOVA test may be repeated many times and remains a valid indicator of attention span and reaction time because there is no learning experience with this test. Therefore, it is ideal for following a patient before, during, and after treatment. Hence, mold exposure can result in toxic

milder than the 14.7-29.4 value in traditional HBOT. Feet of sea water (FSW) is used to indicate either an actual depth, or in this case, a pressure equal to that depth as can be used to describe a hyperbaric chamber. Again, less pressure is applied to the chambers used in this study. The oxygen concentration is mildly elevated from $21 \%$ in air to $24 \%$, yet, is much less than the $100 \%$ oxygen concentration used in traditional chambers

\begin{tabular}{llll}
\hline & Sea level & Mild HBOT & Traditional HBOT \\
\hline ATA & 1.0 & 1.3 & $2.0-3.0$ \\
PSIG & 0 & 4.7 & $14.7-29.4$ \\
FSW & 0 & 11 & $33-66$ \\
Oxygen concentration & $21 \%$ & $24 \%$ & $100 \%$ \\
\hline
\end{tabular}




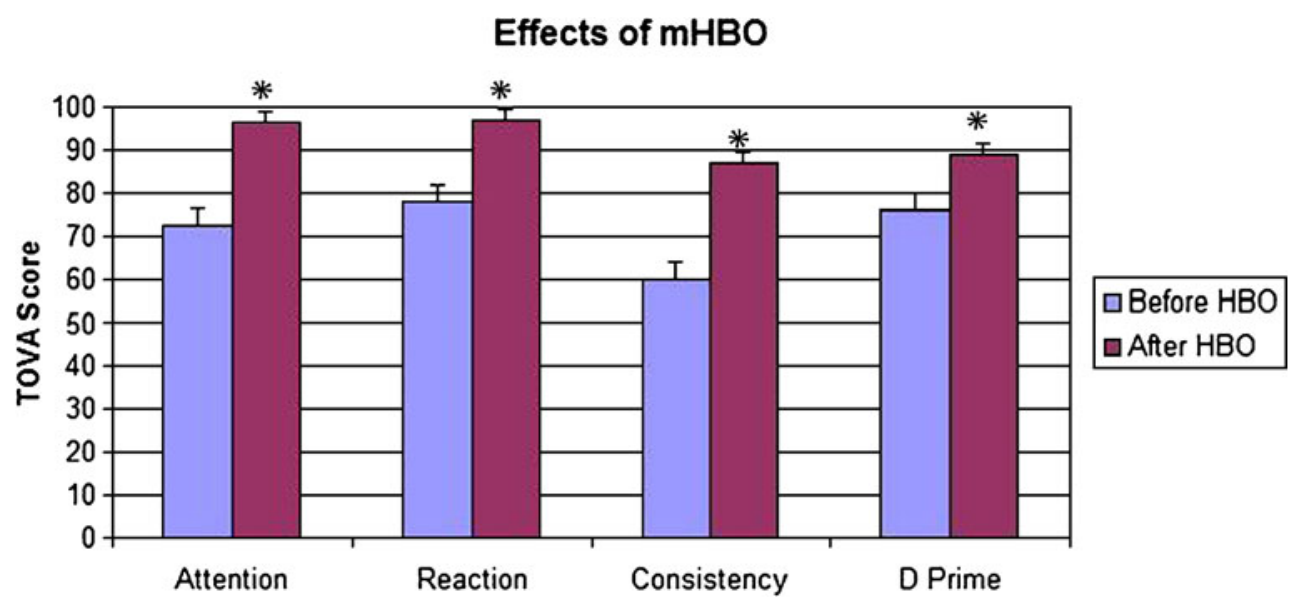

Fig. 1 After ten treatment sessions of mild hyperbaric oxygen treatment (mHBOT), the 15 individuals in the study significantly improved (indicated by ${ }^{*}$ ) in attention span, reaction time, consistency, and an overall D prime score assessed via the Test of Variables of Attention (TOVA). Each subject was compared to his/her own TOVA score before treatment. This figure shows the pooled scores of all 15 subjects before and after all HBOT sessions. A higher score indicates an increase in attention, shortened reaction time, higher consistency, and increased $\mathrm{D}$ prime score. The scale from $0-100$ on the $y$-axis denotes deviation from standard scores exhibited by TOVA controls, where $100=$ no abnormality

with reduced circulation, improving blood flow to areas with arterial blockage. There is a rebound arterial dilation after HBOT, resulting in an increased blood vessel diameter greater than when therapy began, improving blood flow to compromised organs. HBOT stimulates an adaptive increase in superoxide dismutase (SOD), one of the body's principal, internally produced antioxidants and free radical scavengers [22]. Finally, HBOT greatly aids the treatment of infection by enhancing white blood cell action and potentiating germ-killing antibiotics.

The potential mechanism that may underlie the effectiveness of hyperbaric oxygen on ADD symptoms is not clear. HBOT may play a role in the repair or reconstruction of injured lower motor neurons [23] and has been suggested to provide an effective strategy for the prevention and treatment of numerous neurologic handicaps [24].

Stem or progenitor cells have been found in the adult brain in the past decade that can lead to neural regeneration [25], a recovery process that is oxygen-dependent. Capillary density can be increased in the adult mammalian brain [26], aiding nutrient delivery to regenerating tissues. HBOT has been shown to be useful in the treatment of neurologic injury, even if applied in the nonacute period [27, 28].

The results obtained through the current study are preliminary due to the lack of a control group with a possible placebo structure. It is important to take the implications of this small study and implement a controlled, randomized, double-blind experimental study to test the true therapeutic capabilities of hyperbaric oxygen medicine on patients with toxic mold exposure. Control studies with hyperbaric oxygen are very difficult to perform and have been recently discussed by Clarke [29]. 
In a recent multicenter, randomized, double-blind, controlled trial, children with autism who received hyperbaric treatment at 1.3 ATM and $24 \%$ oxygen for 40 hourly sessions had significant improvements in overall functioning, receptive language, social interaction, eye contact, and sensory/cognitive awareness compared to children who received slightly pressurized room air [30]. Other studies have also provided evidence for the hyperbaric treatment of autism [31-35].

While not new, HBOT has only lately begun to gain recognition for the treatment of chronic degenerative health problems related to diabetic gangrene [36], atherosclerosis [37], stroke [38], chronic vascular wound management [39], diabetic foot ulcers [36], wound healing [40], cerebral palsy [41], brain injury [42], multiple sclerosis [43], macular degeneration [44], and many other disorders. Wherever blood flow and oxygen delivery to vital organs are reduced, function and healing can potentially be aided with HBOT.

Memory, reaction time, and visual motor speed assessments were compared before, during, and after hyperbaric oxygen therapy in a case of fetal alcohol syndrome involving a teenage male patient [45]. The authors were able to show that this patient benefited from a short course of low-pressure hyperbaric oxygen therapy, sustained durable cognitive improvements, and continued to exhibit improvement with another short course of treatments [45].

Previous literature has shown HBOT to be effective in improving cerebral palsy using three-dimensional displays of SPECT of the cerebral hemispheres of patients before and after treatment with mHBOT [13]. The computer program used in this study makes a regional quantitative comparison of an individual's SPECT scan with a group of more than 20 controls. Using the same procedure for the 15 patients suffering from toxic injury due to mold exposure, the baseline impairment of perfusion moved into the normal zones after treatment with HBOT. The quantitative aspects of this study are currently being studied.

Low-pressure HBOT is a therapy with an extremely low risk profile and relatively low cost, with potential benefits that seem to be significant and measurable for a condition with few other treatments at our disposal. In this study, 15 adults with symptoms associated with exposure to mold and mycotoxins benefited from a short course of lowpressure HBOT and sustained durable cognitive improvements. Given the implications, these results should receive consideration for broader study.

Conflict of interest statement The authors report no conflicts of interest.

Ethical approval The UCLA IRB approved the consent forms and this study.
Open Access This article is distributed under the terms of the Creative Commons Attribution Noncommercial License which permits any noncommercial use, distribution, and reproduction in any medium, provided the original author(s) and source are credited.

\section{References}

1. Weidenbörner M (2001) Pine nuts: the mycobiota and potential mycotoxins. Can J Microbiol 47:460-463

2. Heuser G, Mena I (1998) NeuroSPECT in neurotoxic chemical exposure: demonstration of long-term functional abnormalities. Toxicol Ind Health 14:813-827

3. Redlich CA, Sparer J, Cullen MR (1997) Sick-building syndrome. Lancet 349:1013-1016

4. Rothman AL, Weintraub MI (1995) The sick building syndrome and mass hysteria. Neurol Clin 13:405-412

5. Anyanwu E, Campbell AW, High W (2002) Brainstem auditory evoked response in adolescents with acoustic mycotic neuroma due to environmental exposure to toxic molds. Int J Adolesc Med Health 14:67-76

6. Gray MR, Thrasher JD, Crago R, Madison RA, Arnold L, Campbell AW et al (2003) Mixed mold mycotoxicosis: immunological changes in humans following exposure in water-damaged buildings. Arch Environ Health 58:410-420

7. Khalili B, Montanaro MT, Bardana EJ Jr (2005) Inhalational mold toxicity: fact or fiction? A clinical review of 50 cases. Ann Allergy Asthma Immunol 95:239-246

8. Anyanwu EC, Campbell AW, Vojdani A (2003) Neurophysiological effects of chronic indoor environmental toxic mold exposure on children. ScientificWorldJournal 3:281-290

9. Anyanwu E, Ehiri J, Akpan AI (2004) Application, effectiveness, and limitations of the electrophysiological diagnosis of neurotoxic effects of chronic environmental mycotoxins in humans. Int $\mathrm{J}$ Adolesc Med Health 16:107-118

10. Wender PH, Wolf LE, Wasserstein J (2001) Adults with ADHD. An overview. Ann N Y Acad Sci 931:1-16

11. Thrasher JD, Heuser G, Broughton A (2002) Immunological abnormalities in humans chronically exposed to chlorpyrifos. Arch Environ Health 57:181-187

12. Vojdani A, Thrasher JD, Madison RA, Gray MR, Heuser G, Campbell AW (2003) Antibodies to molds and satratoxin in individuals exposed in water-damaged buildings. Arch Environ Health 58:421-432

13. Heuser G, Uszler JM (2001) Hyperbaric oxygenation for cerebral palsy. Lancet 357:2053-2054

14. Greenberg LM, Waldman ID (1993) Developmental normative data on the test of variables of attention (T.O.V.A.). J Child Psychol Psychiatry 34:1019-1030

15. Biederman J, Faraone SV, Spencer T, Wilens T, Norman D, Lapey KA et al (1993) Patterns of psychiatric comorbidity, cognition, and psychosocial functioning in adults with attention deficit hyperactivity disorder. Am J Psychiatry 150:1792-1798

16. García-Covarrubias L, Barratt DM, Bartlett R, Van Meter K (2004) Treatment of mucormycosis with adjunctive hyperbaric oxygen: five cases treated at the same institution and review of the literature. Rev Invest Clin 56:51-55

17. Ferguson BJ, Mitchell TG, Moon R, Camporesi EM, Farmer J (1988) Adjunctive hyperbaric oxygen for treatment of rhinocerebral mucormycosis. Rev Infect Dis 10:551-559

18. John BV, Chamilos G, Kontoyiannis DP (2005) Hyperbaric oxygen as an adjunctive treatment for zygomycosis. Clin Microbiol Infect 11:515-517

19. Barratt DM, Van Meter K, Asmar P, Nolan T, Trahan C, GarcíaCovarrubias L et al (2001) Hyperbaric oxygen as an adjunct in 
zygomycosis: randomized controlled trial in a murine model. Antimicrob Agents Chemother 45:3601-3602

20. Gudewicz TM, Mader JT, Davis CP (1987) Combined effects of hyperbaric oxygen and antifungal agents on the growth of Candida albicans. Aviat Space Environ Med 58:673-678

21. Gill AL, Bell CN (2004) Hyperbaric oxygen: its uses, mechanisms of action and outcomes. QJM 97:385-395

22. Kudchodkar BJ, Pierce A, Dory L (2007) Chronic hyperbaric oxygen treatment elicits an anti-oxidant response and attenuates atherosclerosis in apoE knockout mice. Atherosclerosis 193:2835

23. Nakajima M, Kuwabara S, Uchino F, Hirayama K (1994) Enhanced regeneration of terminal axons after hyperbaric oxygen therapy in a patient resembling progressive postpoliomyelitis muscular atrophy. Rinsho Shinkeigaku 34:48-51

24. Calvert JW, Yin W, Patel M, Badr A, Mychaskiw G, Parent AD et al (2002) Hyperbaric oxygenation prevented brain injury induced by hypoxia-ischemia in a neonatal rat model. Brain Res 951:1-8

25. Steindler DA, Pincus DW (2002) Stem cells and neuropoiesis in the adult human brain. Lancet 359:1047-1054

26. Harik SI, Behmand RA, LaManna JC (1994) Hypoxia increases glucose transport at blood-brain barrier in rats. J Appl Physiol 77:896-901

27. Ersanli D, Yildiz S, Togrol E, Ay H, Qyrdedi T (2004) Visual loss as a late complication of carbon monoxide poisoning and its successful treatment with hyperbaric oxygen therapy. Swiss Med Wkly 134:650-655

28. Ziser A, Shupak A, Halpern P, Gozal D, Melamed Y (1984) Delayed hyperbaric oxygen treatment for acute carbon monoxide poisoning. Br Med J (Clin Res Ed) 289:960

29. Clarke D (2009) Effective patient blinding during hyperbaric trials. Undersea Hyperb Med 36:13-17

30. Rossignol DA, Rossignol LW, Smith S, Schneider C, Logerquist S, Usman A et al (2009) Hyperbaric treatment for children with autism: a multicenter, randomized, double-blind, controlled trial. BMC Pediatr 9:21

31. Yildiz S, Aktas S, Uzun G (2008) Hyperbaric oxygen therapy in autism: is there evidence? Undersea Hyperb Med 35:453-455

32. Chungpaibulpatana J, Sumpatanarax T, Thadakul N, Chantharatreerat C, Konkaew M, Aroonlimsawas M (2008) Hyperbaric oxygen therapy in Thai autistic children. J Med Assoc Thai 91:1232-1238
33. Rossignol DA, Rossignol LW, James SJ, Melnyk S, Mumper E (2007) The effects of hyperbaric oxygen therapy on oxidative stress, inflammation, and symptoms in children with autism: an open-label pilot study. BMC Pediatr 7:36

34. Rossignol DA (2007) Hyperbaric oxygen therapy might improve certain pathophysiological findings in autism. Med Hypotheses 68:1208-1227

35. Rossignol DA, Rossignol LW (2006) Hyperbaric oxygen therapy may improve symptoms in autistic children. Med Hypotheses 67:216-228

36. Faglia E, Favales F, Aldeghi A, Calia P, Quarantiello A, Oriani G et al (1996) Adjunctive systemic hyperbaric oxygen therapy in treatment of severe prevalently ischemic diabetic foot ulcer. A randomized study. Diabetes Care 19:1338-1343

37. Kudchodkar BJ, Wilson J, Lacko A, Dory L (2000) Hyperbaric oxygen reduces the progression and accelerates the regression of atherosclerosis in rabbits. Arterioscler Thromb Vasc Biol 20:1637-1643

38. Nighoghossian N, Trouillas P, Adeleine P, Salord F (1995) Hyperbaric oxygen in the treatment of acute ischemic stroke. A double-blind pilot study. Stroke 26:1369-1372

39. Wattel F, Mathieu D, Coget JM, Billard V (1990) Hyperbaric oxygen therapy in chronic vascular wound management. Angiology 41:59-65

40. Fabian TS, Kaufman HJ, Lett ED, Thomas JB, Rawl DK, Lewis PL et al (2000) The evaluation of subatmospheric pressure and hyperbaric oxygen in ischemic full-thickness wound healing. Am Surg 66:1136-1143

41. Papazian O, Alfonso I (2003) Hyperbaric oxygen treatment for children with cerebral palsy. Rev Neurol 37:359-364

42. Chuba PJ, Aronin P, Bhambhani K, Eichenhorn M, Zamarano L, Cianci $P$ et al (1997) Hyperbaric oxygen therapy for radiationinduced brain injury in children. Cancer 80:2005-2012

43. Fischer BH, Marks M, Reich T (1983) Hyperbaric-oxygen treatment of multiple sclerosis. A randomized, placebocontrolled, double-blind study. N Engl J Med 308:181-186

44. Ghazi NG, Jabbour NM, De La Cruz ZC, Green WR (2001) Clinicopathologic studies of age-related macular degeneration with classic subfoveal choroidal neovascularization treated with photodynamic therapy. Retina 21:478-486

45. Stoller KP (2005) Quantification of neurocognitive changes before, during, and after hyperbaric oxygen therapy in a case of fetal alcohol syndrome. Pediatrics 116:e586-e591 